\title{
Divulgar para Atrair, Motivar para Manter
}

\author{
Sílvia Amélia Bim, Marília A. Amaral, Nádia P. Kozievitch, Maria Claudia F. P. \\ Emer, Mariângela O. G. Setti, Luiz A. Pellison, Luiz E. Merkle
}

Departamento Acadêmico de Informática, Universidade Tecnológica Federal do Paraná

(UTFPR) - Curitiba, PR - Brasil

\{sabim, mariliaa, nadia, mcemer, mariangelasetti, pelisson, merkle\} @utfpr.edu.br

\begin{abstract}
The increasing masculinization of Computing is a global concern. The scenario requires diverse actions among all the people involved. This article presents some of the initiatives carried out in the context of an extension project which aims do diversify the dimensions of Computing, in order to both diversify the participation and attract more women to the area. We also present actions carried out to motivate current students of Information Systems and Computer Engineering degrees of a Brazilian public university, in order to contribute to lower the drop-out rates of women in these degrees.
\end{abstract}

Resumo. A crescente masculinização da área de Computação é uma preocupação mundial. O cenário exige ações diversificadas com todas as pessoas envolvidas. Neste artigo apresentamos algumas das iniciativas realizadas no contexto de um projeto de extensão que visa diversificar as dimensões da Computação com o objetivo de também diversificar a participação, atraindo mais mulheres para área. Além disso, também apresentamos algumas ações desenvolvidas para motivar as atuais estudantes dos cursos de Sistemas de Informação e Engenharia da Computação de uma universidade pública brasileira, de forma a contribuir para um menor indice de evasão de mulheres nestes cursos.

\section{Introdução}

Desde os anos 80 observa-se um decréscimo da presença de mulheres dentre os/as concluintes dos cursos da área de Computação no Brasil [Oliveira et. al. 2014]. Para tentar reverter este cenário, diversas ações estão sendo realizadas no país. Uma iniciativa é o Programa Meninas Digitais da Sociedade Brasileira de Computação (SBC) [Maciel e Bim, 2016] que incentiva e apoia a criação de projetos que divulguem a Computação para estudantes mulheres nos ensinos médio e fundamental. Anualmente, este Programa também realiza o Fórum Meninas Digitais, incluso na programação do WIT - Women in Information Technology, evento base do Congresso da Sociedade Brasileira de Computação.

Embora a SBC tenha iniciativas que buscam promover a maior participação de mulheres na Computação ainda há muito a ser feito. Em 38 anos de existência, a SBC só teve uma presidente mulher - Claudia Bauzer de Medeiros (2003 a 2007 - duas gestões) e uma vice presidente mulher, Thais Vasconcelos Batista (2016 a 2018 - atual 
gestão). Na gestão atual, de oito diretorias, três $(37,5 \%)$ são dirigidas por mulheres. Em relação às 36 comissões especiais da SBC, em 2015, 10 (27\%) são coordenadas por mulheres.

Entretanto, além de programas de incentivo e atração de novos talentos para a Computação, neste caso mulheres, também são urgentes ações que viabilizem a permanência de mulheres profissionais na área e incentivem as mulheres a ocuparem posições de liderança nos contextos onde atuam.

Este artigo apresenta o Projeto Emíli@s - Armação em Bits ${ }^{1}$, suas ações e resultados obtidos desde a sua criação. Tal projeto realiza ações para divulgar a computação para estudantes do ensino médio de escolas públicas de Curitiba. Além disto, promove a integração de alunas de pós-graduação com as alunas de graduação, realiza atividades para incrementar a permanência de mulheres nos cursos de Sistemas de Informação e Engenharia de Computação e, consequentemente, diminuir os índices de evasão. O Emíli@s também organiza encontros para docentes e estudantes, de ambos os sexos, para discutir questões de gênero, pois acredita-se que é necessário ampliar e aprofundar as reflexões sobre o tema Mulheres na Computação, a fim de que seja possível, por meio da colaboração de todas e todos, uma diversificação da participação na área.

O artigo é estruturado como segue. Na seção seguinte, o histórico do projeto é apresentado. As Seções 3 e 4 abordam ações de divulgação e ações de motivação, respectivamente. As considerações finais são feitas na Seção 5 que encerra o artigo.

\section{O projeto Emíli@s - Armação em Bits}

O Projeto Emíli@s - Armação em Bits é uma iniciativa do Departamento Acadêmico de Informática (DAINF) da UTFPR - Universidade Tecnológica Federal do Paraná, câmpus Curitiba. A primeira ação do projeto foi realizada no dia 08 de março de 2013. Neste primeiro ano o projeto foi cadastrado na universidade como projeto de pesquisa. A partir de 2014 o projeto foi cadastrado como projeto de extensão, sendo que no ano de 2014 teve o apoio financeiro do edital MCTI/CNPq/SPMPR/Petrobras n ${ }^{\circ} 18 / 2013$ Meninas e Jovens Fazendo Ciências Exatas, Engenharias e Computação. Em 2016 o projeto conta com a colaboração de um casal de docentes da UTFPR-Campo Mourão, que com a colaboração de estudantes realiza ações no campus onde atuam.

O nome do projeto é inspirado na personagem de Monteiro Lobato ${ }^{2}$, Emília $^{3}$. O objetivo é reconhecer a voz das mulheres na Computação, as atuais e futuras. Entende-se que é necessário incentivá-las a armar, propor e resolver problemas da sociedade, usando a Computação, no sentido clássico de estruturar, aparelhar, abastar. Por isto, armação em bits.

Ao longo de sua história, o projeto teve a participação direta de oito docentes, cinco mulheres e três homens, na equipe que coordena as ações. Em 2014, foram alocadas bolsas, com os recursos do edital citado, a uma estudante do curso de Sistemas de Informação da UTFPR, e a quatro estudantes do ensino médio, e à coordenadora

\footnotetext{
${ }^{1}$ http://emilias.dainf.ct.utfpr.edu.br/

${ }^{2}$ https://pt.wikipedia.org/wiki/Monteiro_Lobato

${ }^{3}$ https://pt.wikipedia.org/wiki/Emília_(personagem)
} 
pedagógica da instituição parceira, o Colégio Estadual Dr. Xavier da Silva. Em todos os anos vários e várias estudantes voluntários(as) colaboraram com as ações, sendo em sua maioria integrantes de dois Programas de Educação Tutorial (PET) existentes no DAINF: PETCoCE ${ }^{4}$ - PET Computando Culturas em Equidade e PETECO ${ }^{5}$ - PET de Engenharia de Computação. Outras ações foram realizadas em parceria com o Compute você Mesm@: Conexões de saberes e fazeres para inclusão digital (projeto de extensão do DAINF), outras ainda, contaram com a participação de estudantes do Programa de Pós-graduação em Computação Aplicada (PPGCA/ DAINF).

\section{Ações de divulgação}

Ao longo de três anos e meio o projeto Emíli@s realizou diversas ações de divulgação da área de Computação. Buscaram-se, com estas ações, mostrar diversas dimensões da Computação a fim de divulgar as inúmeras possibilidades de atuação na área. Enfatizaram-se as demandas e as vantagens de profissionais com perfis diversificados, de modo a melhor compreender diferentes modos de computar, de diferentes pontos de vista. Como resultado destas ações, espera-se que os esteriótipo profissional em Computação possa ser desmistificado e criticado.

Uma das ações foi a realização de uma palestra para docentes da escola parceira - Colégio Estadual Dr. Xavier da Silva. A coordenadora do projeto Emíli@s apresentou ao corpo docente, durante a semana de formação, o cenário atual da Computação e a necessidade de atrair mais mulheres para a área.

Quatro oficinas práticas foram ofertadas para as estudantes do ensino médio da escola parceira. Estudantes de outras escolas também participaram:

- Oficina prática de Interação Humano-Computador [Amaral et al, 2015];

- Oficina prática de Banco de Dados [Rodriguez et al, 2016];

- Oficina para desenvolvimento de aplicativos Android usando App Inventor;

- Oficina de Webdesign Básico.

Mulheres profissionais, com diferentes perfis (formação e atuação), foram convidadas a compartilharem suas experiências fazendo palestras. Tais palestras aconteceram no próprio ambiente universitário. Uma delas também foi reapresentada na escola parceira.

Por fim, foram organizados eventos em diferentes épocas do ano. "Debatendo Gênero" foi realizado em parceria com outros projetos da universidade. A programação consistiu em duas mesas redondas com a participação de estudantes, docentes e profissionais buscando apresentar diferentes pontos de vista e experiências sobre o tema. Em 2014 e 2015, nos meses de outubro, foram realizadas atividades para celebrar o Ada Lovelace Day [Bim, 2016]. Nos anos de 2013, 2014 e 2016 o projeto organizou atividades para o Dia Internacional da Mulher. Em 2016, por exemplo, houve a exibição do filme Code Girl ${ }^{6}$, palestras com profissionais da área de Engenharia da Computação,

\footnotetext{
${ }^{4}$ http://www.dainf.ct.utfpr.edu.br/petcoce/

${ }^{5} \mathrm{http}$ ://dainf.ct.utfpr.edu.br/peteco/

${ }^{6} \mathrm{http}: / /$ www.codegirlmovie.com/
} 
depoimento da única estudante brasileira que participou da Grace Hopper Conference em 2015 e uma palestra sobre feminismo.

\section{Ações de motivação}

Para ter o apoio das estudantes dos cursos de Sistemas de Informação, Engenharia da Computação e da pós-graduação em Computação Aplicada, nas ações do projeto Emíli@s identificou-se a necessidade de conhecer a experiência destas estudantes nos cursos. Para isto foram realizados edições de dois eventos.

O Pipocando Ideias foi uma ação realizada para promover o encontro das estudantes dos cursos graduação e pós-graduação, assim como discutir ações para recepcionar calouras destes cursos e ações voltadas a estudantes do ensino médio.

O Bate Papo de Emíli@s teve o objetivo de proporcionar uma oportunidade de encontro entre estudantes dos cursos e algumas docentes, de modo que pudessem compartilhar suas experiências no contexto acadêmico.

\section{Considerações finais}

É possível identificar resultados de diferentes naturezas a partir das ações do Projeto Emíli@s - Armação em Bits. Um destes resultados foi a realização de duas pesquisas sobre a presença de mulheres no curso de Sistemas de Informação, realizadas por duas duplas de estudantes mulheres, no contexto da disciplina de Metodologia de Pesquisa, deste mesmo curso. A partir destas pesquisas, foi criada a Diretoria Feminina do Centro Acadêmico de BSI que organizou diversas atividades de integração entre as estudantes mulheres do curso.

Algumas reflexões sobre gênero, que já existiam no DAINF antes da criação do Projeto Emíli@s, contribuíram claramente na reformulação curricular do curso de Sistemas de Informação [Setti et al, 2014].

As avaliações feitas após as atividades são positivas. A parceria com a escola se renova anualmente e sempre há interesse das estudantes em participar das ações propostas. A discussão sobre gênero está presente no cotidiano do Departamento e percebe-se um crescente engajamento dos(as) atuais estudantes nos questionamentos feitos durante os eventos e fora deles. Os resultados parciais alcançados até o momento são positivos.

\section{Referências}

Amaral, M.A., Bim, S.A., Boscarioli, C., Maciel, C. (2015) "Introducing Computer Science to Brazilian Girls in Elementary School through HCI Concepts" In: HCI International 2015 - Session: Women in DUXU, Los Angeles, CA, Lecture Notes in Computer Science - Design, User Experience, and Usability: Users and Interactions. Proceedings, Part II, 2015. v. 9187. p. 141-152.

Bim, S. A. Ada Lovelace - falando sobre gênero nas aulas de Algoritmos. In: Computer on the Beach, 2016, Florianópolis. Anais [do] Computer on the Beach, 2016. p. 300-309. 
Maciel, C., Bim, S. A. Programa Meninas Digitais - ações para divulgar a Computação para meninas do ensino médio. In: Computer on the Beach, 2016, Florianópolis. Anais [do] Computer on the Beach, 2016. p. 327-336.

Rodriguez, J. J. ; Kozievitch, N. P. ; Bim, S. A. ; Setti, M. G. ; Emer, M. C. F. P. ; Amaral, M. A. . Uma Proposta para Apresentar a Computação - Banco de Dados no Ensino Médio para o Público Feminino. In: Escola Regional de Banco de Dados SBC, 2016, Londrina - PR. XII Escola Regional de Banco de Dados - Tema Data Science, 2016. v. 1. p. 155-158.

Oliveira, A.C., Moro, M.M., Prates, R.O. (2014) "Perfil Feminino em Computação: Análise Inicial” In: XXII Workshop sobre Educação em Computação, Brasília, DF, p. $1465-1474$.

Setti, M.G., Emer, M.C.F.P., Amaral, M.A., Merkle, L.E. e Gonçalves, M.M. (2014) "Proposta de Flexibilização Curricular do Curso de Sistemas de Informação ofertado pela UTFPR-Curitiba", In: XXII Workshop sobre Educação em Computação, Brasília, DF, p. 1366-1375. 